\title{
Some Issues on the Design of a Low-Cost Autonomous Underwater Vehicle with an Intelligent Dynamic Mission Planner for Pipeline and Cable Tracking
}

\author{
Gerardo Gabriel Acosta1-2, Hugo Curti³, \\ Oscar Calvo Ibáñez ${ }^{4}$ and Silvano Rossi ${ }^{1}$ \\ ${ }^{1}$ Grupo INTELYMEC - Univ. Nac. del Centro de la Prov. de Buenos Aires. \\ ${ }^{2}$ Consejo Nacional de Investigaciones Científicas y Técnicas CONICET. \\ ${ }^{3}$ Grupo INTIA - Univ. Nac. del Centro de la Prov. de Buenos Aires. \\ ${ }^{4}$ Grupo de Tecnología Electrónica - Univ. de las Islas Baleares. \\ 1,2,3Argentina \\ ${ }^{4}$ España
}

\section{Introduction}

The main goal of this chapter is to introduce to the design and working hypotheses for the construction of a low-cost Autonomous Underwater Vehicle (AUV) devoted to pipeline and cable inspections. Underwater inspection is mainly necessary on account of the periodic surveys for the preventive maintenance of submarine infrastructure in the off-shore industries. The advantages of doing them with AUVs instead of Remote Operated Vehicles (ROV) or Towed Unmanned Devices (TUD) are low costs and better data quality in the inspection missions. The vehicle presented in this chapter was thought as an experimental platform to test multiple algorithms and to develop new technology, mainly using artificial intelligence techniques. In this sense, its modular architecture tackles the four main needs that should be solved in an autonomous vehicle to perform useful tasks in an unknown and strongly disturbing environment like the underwater world. They are: a) a robust control system to manage nonlinearities and disturbances, b) a precise guidance system to avoid unnecessary time and thus energy consumption, c) an accurate navigation system to determine self and target's positions, and d) an intelligent dynamic planner proposing the best possible trajectories and actions to successfully reach the mission objectives, based on decisions taken without human intervention. In a comparison with a biological being, the autonomous robot also need some kind of controlled muscles and forces to move, self perception and notion of the surroundings, and a brain to plan actions and movements.

In this work, different approaches for all of the aforementioned systems will be presented and thoroughly analyzed at the light of experimental evidence and author's experience in mobile robots (Fernández León et al., 2008). These experiments comprise computer simulations, hardware in the loop simulation as well as sea trials with the low-cost prototype described in the sequel, which is expected to navigate in the sea up to $100 \mathrm{~m}$ of depth. 
This chapter is organized as follows. A brief introduction to the application problem and a context for autonomous underwater vehicles will be presented in section 2 . The hardware and software architecture for the AUV prototype will be discussed in sections 3 . Section 4 will be devoted to experimental results analysis, and final conclusions of the whole chapter and future works will be given in the final sections.

\section{Autonomous underwater vehicles for pipeline and cable inspections}

\subsection{Overview}

The current need of energy transport such as electricity, petroleum and gas have provoked an increasing amount of underwater infrastructure such as cables and pipelines. In order to maintain these infrastructures with a suitable degree of safety and reliability, periodic inspections for preventive maintenance are necessary.

Damages in the submarine pipelines due to suspended (free-span) sections, craft anchorages or fishing activities can cause a strong environmental impact and to cut off certain critical supplies or communication lines. Although leakages of a submarine pipeline are not frequent, the consequences of an eventual spillage to the environment may be severe and irreversible. On the other hand, a greater demand of inspections and preventive maintenance are necessary due to pipeline ageing. In addition, many parts of the geographical areas with submarine infrastructure are located in deep waters $(500-3500 \mathrm{~m}$.), constituting another challenge for the current technology.

A similar situation is observed in the case of preventive maintenance of submarine cables, because there is not an international standard to carry out it. A great reason to introduce these periodic surveys for the preventive maintenance is to reduce the repair time and, therefore, the profit losses due to the impossibility of information transmission through the cable. In addition, the spillages of pollutants of the damaged electrical submarine cables have also a dramatic impact in the fragile marine environment. To minimize it, it is necessary to urgently locate any possible damage, in order to take the necessary precautions for avoiding the pollution. Therefore, the above-mentioned maintenance also includes the recognition (and the corresponding decision about navigation behaviour of the submarine robot) of wastes located in the proximity of the inspected object. Thus, the shape recognition of fishing nets, rocks, mines, anchors, and other debris, should be also considered.

There are then two main motivations for preventive maintenance: to avoid infrastructure damage and for ecosystem preservation, which are closely related. Based on the previous observations, it is clear that one of the most outstanding applications for AUVs is pipeline and cable tracking for maintenance purposes. This explains the increasing interest on commercial exploitation of periodical underwater inspections.

Currently these inspections are done with ROV or TUD as mentioned, but these approaches have two basic drawbacks when compared to an AUV without a physical link to the surface: a) the lower quality of acquired data due to umbilical perturbation over position, and b) the higher cost to be invested in the ship and its crew each time that an inspection has to be undertaken. These two unwanted characteristics become enhanced, as the surveys depths are greater. For instance, offshore petroleum exploitation is being shifted to deeper waters as the resource is becoming scant. In contrast, AUVs allow a smother and faster navigation (over the typical three quarter knots of ROVs), and then a more reliable data acquisition is obtained. In fact, an AUV can reach positions in global coordinates and navigate in autonomous way with low position error, and is able to follow certain sensors readings 
considering them for planning the desired and possible trajectory to be tracked the vehicle's control systems. However, there are other limitations like the pressure that the submarine can stand when the depth increases and the endurance in terms of battery power. These are still open problems from standpoint of new materials.

During the last years successful trials have been done with AUVs applied to cable and pipeline tracking. Among them, the paradigmatic Twin-burger 2, guided by cam images (Balasuriya \& Ura, 1998), although for deep and opaque waters it is preferable to use sonar or a fusion of many sensors like in RAIS (Antonelli et al., 2001). Also the EU funded AUTOTRACKER Project, in which the authors participated, was thought to show that the current technology is mature enough to face this autonomous underwater pipelines and cables inspections in deep water up to three thousand meters. Some reports on preliminary successful results may be found in (Evans et al., 2003) and (Acosta et al., 2005). They were the antecedents for the AUVI prototype, also supported by the EU and the University of Balearic Islands, (Acosta et al., 2006), and (Acosta et al., 2007). The AUVI was constructed mainly to test computational intelligence algorithms for planning and replannig of vehicle's trajectories and tasks, and is the ancestor of the current prototype ICTIOBOT.

\subsection{AUV general architecture for target tracking}

The necessary building blocks for an AUV devoted to pipeline and cable tracking are depicted in figure 1, and explained in the following paragraphs.

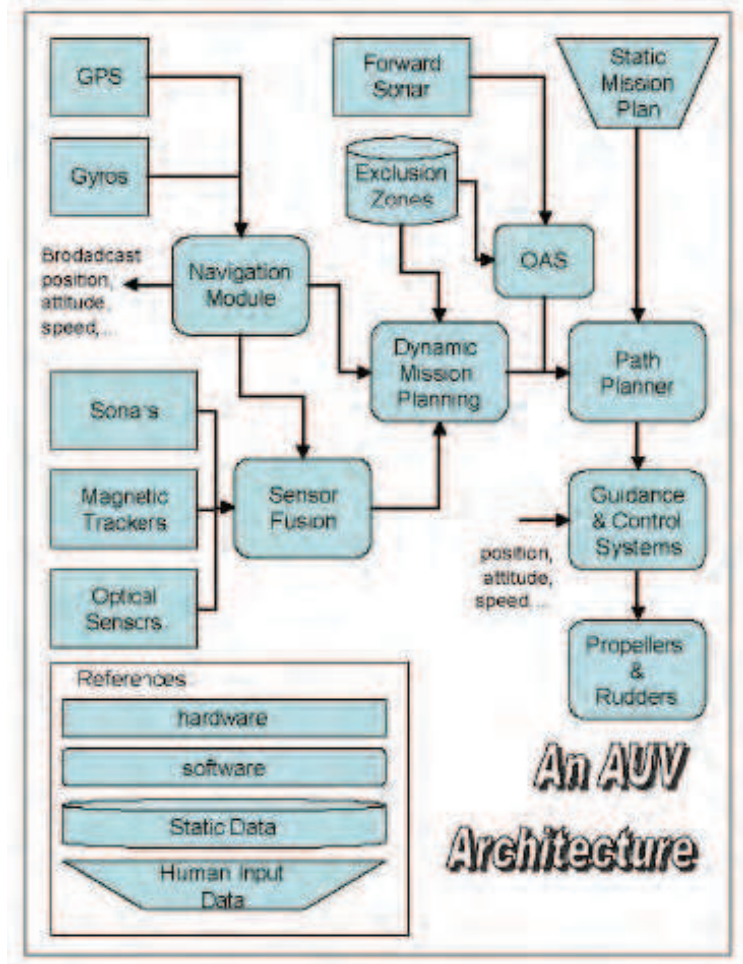

Fig. 1. Building blocks for an AUV software/hardware architecture 
The navigation module is usually referred to the on board sensory systems. It comprises the data fusion necessary to locate precisely in 3D the AUV rigid body and the target position. The usual components within the navigation system are a global position system (GPS), an inertial navigation system (INS), a compass, a depth sensor, and others. Thus, the navigation system provides the dynamic mission planner system, the guidance system and the control system with accurate data to achieve their objectives.

The guidance module is frequently associated to a low-level trajectory generation. When the waypoints for the robot are defined, a trajectory to reach them is necessary in order to feed the controllers set points.

The control module is regarded to the feedback loops allowing the vehicle to describe the trajectory as close as possible to the proposed path given by the guidance module. In effect, assuming that the navigation system yields a clear perception of the AUV's positions, speeds and headings, and the guidance system gives a suitable trajectory to reach a waypoint, there is still remaining a module capable of maintaining the vehicle as close as possible to the prefixed trajectory. Established in this way, the problem to solve at this stage is a control problem to command the vehicle actuators (propellers, rudders and pumps).

A mission planner, according to the robot's application, is also necessary to accomplish the task in an autonomous way. A key component of the mission plan is the path planning. Special sensor acquisitions (snapshots, videos, water samples, and others) or special actions (debris grasping) may also be considered within a mission planner. The mission plan consists of two kinds of objectives: long term static objectives and short term changing ones. The first ones, are given beforehand, in a rigid way through a human-machine interface, and then conform a Static Mission Planning, (SMP). The short term objectives can be changed on-line, and constitute the Dynamic Mission Planning (DMP), and varies as the vehicle movement progresses in the real world. This is also known as mission replanning, in response, for instance, to different obstacles to be avoided, based on data from a forward looking sonar and the pipeline or cable position. The artificial intelligence based DMP is a core development in the AUV presented in this article, so it will be explained in more detail in a following section.

When the underwater installation is constructed, legacy data (LD) are archived containing pipeline's or cable's positions, depths, a corridor width and forbidden zones. They should be on-line accessed by the DMP and the obstacle avoidance system (OAS).

Pipeline trajectory may be estimated from special sensors like a multi-beam echo sounder (MBE), a side-scan sonar (SSS), a magnetic tracker (MAG), a DIDSON sonar (DID), cameras (CAM) and others. This information is combined in a sensor fusion module (SFM) yielding a position and direction estimate of the target to be inspected. From these data, the DMP is able to decide a trajectory according to different situations like searching a pipeline, following it, navigating closer to it, or recognizing other objects surrounding it. This desired trajectory is defined as a collection of four (4) waypoints to be reached by the vehicle.

The Path Planner in this architecture only decides if the desired trajectory given by the DMP is possible or not, according to the outcome of the OAS. Then the waypoints belonging to the desired and possible trajectory are inputs to the guidance module.

The Obstacle Avoidance System (OAS) receives data from a forward-looking sonar (FLS). When an obstacle is detected, a near possible waypoint is proposed to correct the desired trajectory from the DMP. 


\subsection{An overview of tracking methods}

The most common methods to track submarine infrastructures are based on magnetic and electromagnetic principles. Robots like ROVs or remote operated towed vehicles are capable of transmitting signals in such a way that the operator takes advantage of them to control its movement by means of a joystick.

In addition to magnetic tracking methods, the operator can use a video camera to prevent seabed collisions. Nevertheless, this method is limited by the poor visibility of the water and can be improved by means of obstacles detection on the basis of acoustic image systems, as already tested in the AUTOTRACKER project (Evans et al., 2003), (Acosta et al., 2005).

The movement estimation and pattern shape recognition from video sequences have received a great attention on behalf of the computer vision community, and several solutions for calibrated and uncalibrated cameras have been proposed in the last years. The current methods use estimations based on optical flow techniques (Barron et al., 1994), that is, the instantaneous velocity field of every image pixel, or in characteristics trackers (Shi \& Tomasi, 1994), who resort to algorithms looking for special elements of the image and they follow them through the whole sequence.

Some existing methods for characteristics tracking seem to be sufficiently solid and mature to support movement estimation and shape recognition in a reliable manner, without an intrinsic computational load associated with optical flow techniques (Rives et al., 1986), (Looney, 1997). However, still it is necessary to investigate how to adapt the existing solutions to the underwater images, since many of the suppositions commonly assumed in the air are violated. For instance, the lightning is not static and independent from the observer movement.

Thus, sonar data processing research has focused in the objects detection, its classification, the obstacle avoidance, and the navigation based on the terrain. Forward-looking sonars (FLS) with mechanical sweep provide of richer information, but they need the correction of the movement using navigation information from the vehicle that transports it. The multibeam MBEs are bigger than the FLS and capable of providing several updates of the image frames. From MBE and by means of simple methods of image processing it is possible to extract useful information of the pipeline or cable for its tracking. Nevertheless, the great challenge is still to reduce the false alarms relation using multiple hypotheses between frame and frame for the tracking.

There is another open problem related to the capability of detecting and classifying different objects from those that are under inspection, employing the same information from the sonars. Likewise, when the objective under study gets lost, the search strategies and reacquisition can be optimized to minimize the search time of the beginning of mission point, or the location, in the future, of targets of different types and shapes. For instance, applying variants of "Random Walk" and "Lévy Fligth" models (Bartumeus et al., 2002).

Both the FLS and the video sensors are commonly available in the submarine commercial vehicles, but they are used by the human pilots as navigation and decision sensors. For its utilization in an AUV, this sensor data must be processed on-line and in real time, and used to provide the input to the control systems in order to carry out a correct tracking and obstacle avoidance. Though there are laboratory systems that do this currently, it must be carefully demonstrated the practical validation in a real system working in the seabed. The state of the art has been demonstrated inside the European programs Esprit III and MAST III, in which ROVs were used with general-purpose computers calculating in the surface. 
The results of these programs have been promissory, indicating that would be opportune to consider fixing these systems directly in the vehicle, which would be obviously an AUV.

\section{The low-cost prototype ICTIOBOT}

The general objective of the work described in this chapter was to design and to develop cost-efficient technology for the inspection of pipelines and submarine cables in maritime infrastructures, including the development of an autonomous and safe navigation system for a submarine prototype. With this prototype it will also be possible to make pattern recognition from sonar's data to detect debris in the proximity of the target to be tracked.

The design considerations and proposed solutions as well as a description of the hardware employed, the sensors in the payload, and the DMP based on an expert system are presented in this section.

The specific aims of this development were the following ones:

- To develop a software module to provide the desired trajectory for an AUV devoted to submarine pipeline and cable tracking with the purpose of inspecting them, resorting to artificial intelligence techniques, particularly knowledge based systems (KBS) and artificial neural networks (ANN). The initial point was the EN4AUV (Expert Navigation for Autonomous Underwater Vehicle) in (Acosta et al., 2003). This module will be more thoroughly described in section 3.5.

- To assemble a low-cost prototype, including a MBE sonar, a SSS sonar, a GPS, an INS, and an industrial PC type as processing unit.

- To develop a pattern recognition system based on artificial neural networks for classifying objects using the data provided by the sonars.

- To validate the resulting prototype by means of its utilization in the sea for carrying out inspections of pipelines and submarine cables to a depth not bigger than $100 \mathrm{~m}$.

\subsection{Working hypotheses}

A modular philosophy was employed in the design and development of the low-cost AUV prototype because several modules that were previously tested in other applications, were reused here. In particular, there is a great interest on knowledge reuse through the generalization in an ontology for mobile robots navigations. In this manner, the design of a knowledge-based DMP easily ported from one application to another is an essential point to research and test within this undergoing project. To accomplish this goal, some working hypotheses were considered:

- The hardware technology is able to deal with pipeline and cable tracking in submarine environments.

- The KBS approach is sufficiently robust for managing the decision process in the realtime planning and replannig of the better trajectory in complex environments.

- It is possible to detect and to recognize different shapes in the seabed from acoustic images by means of the utilization of ANN, and this is much more efficient than using an optical camera for the dark waters of the Argentinean Sea.

- The whole technology and the knowledge base already developed in previous projects can be reused in the ICTIOBOT prototype, for researching in planning, guiding, controlling and navigating algorithms, mainly based in computational intelligence approaches. 
- The prototype should be of low cost not only regarding the effective cost of the prototype but also the operations costs of each sea trial.

\subsection{ICTIOBOT's architecture}

The modular architecture of the prototype is based on the aims and working hypotheses previously described, and it is presented in figure 2, showing the basic modules of any autonomous robot, that is, the mission planner planner, guidance, control, and navigation systems.

The DMP system comprises the EN4AUV knowledge based system and an OAS, based on evolutionary robotic paradigm developed for terrestrial applications (Fernández León, et al. 2004). Both modules, jointly with the guidance and control systems, and the pattern recognition system run in a low consumption on-board PC.

The navigation system is constituted by a compass, a GPS (for navigating in the surface and re-initialization of accumulated errors), an inertial navigation system, and a depth sensor. They are the input of a sensor fusion module that determines the position in absolute coordinates, the attitude and the depth of the robot location.

The other sensors in the payload are a MBE for target tracking and a FLS for obstacle perception. A SSS and a MAG for target searching and tracking will be added in future stages of the project.

Every module has an input-output data flow based on messages put on a UDP channel, and broadcasted for the remaining ones, using TCP/IP protocol. The operating system is GNU Linux running in an industrial $\mathrm{PC}$, and the preponderant programming language is $\mathrm{C}++$.
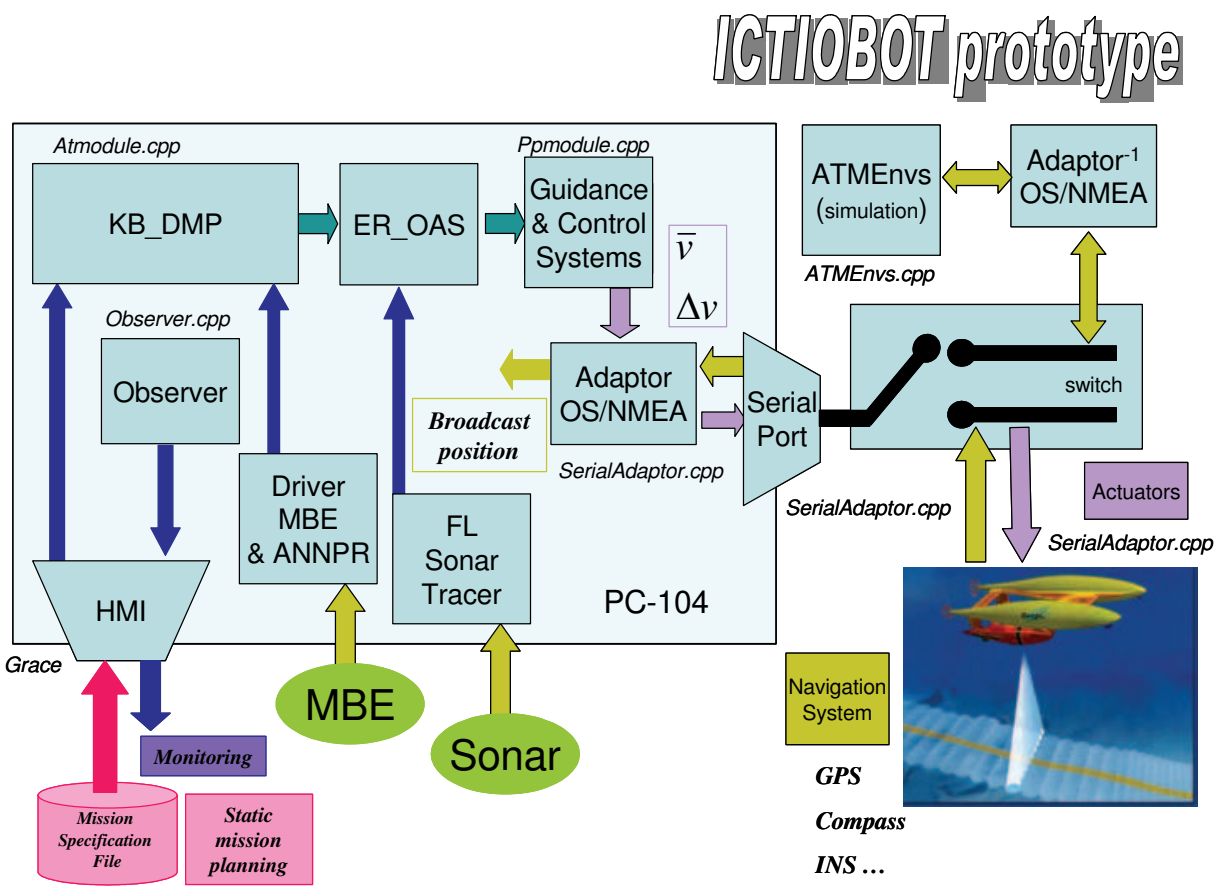

Fig. 2. Modular architecture for the ICTIOBOT prototype 


\subsection{Hardware architecture}

The AUV hardware is composed of two main processor boards: a low level and a high level electronics. The low level electronic is based on the iCm4011 microcontroller development board from Ingenia that uses a dsPIC 30F4011 as main processor. This board provides I2C and RS232 interfaces for communicating with the sensors and the high level processor unit. The high level board is an industrial PC based on Intel X86 microprocessor. This is the environment where most of the planning and controlling software resides. Figure 3 shows, in schematic way, the on-board electronics for the low-coast AUV prototype.

The microcontroller board gets the position either from the GPS when surfaced, or from an accelerometer, integrating both $\mathrm{x}$ and $\mathrm{y}$ readings. From this data and the reading data provided by the depth meter, the microcontroller board is capable to provide the $(x, y, z)$ triplet necessary for the proper position. The GPS sends its data using RS232 streams according to a format specified by the National Marine Electronics Association (NMEA). The accelerometer is based on the Analog Devices ADXL 202, dual axis accelerometer, constituting an inexpensive device that measures accelerations with a full-scale range of $\pm \mathrm{g}$. This sensor outputs are analog voltages or digital signals whose duty cycles, that is, the ratio of pulse width to period, are proportional to acceleration. The duty cycle outputs can be directly measured by the microprocessor.

\section{Hardware}

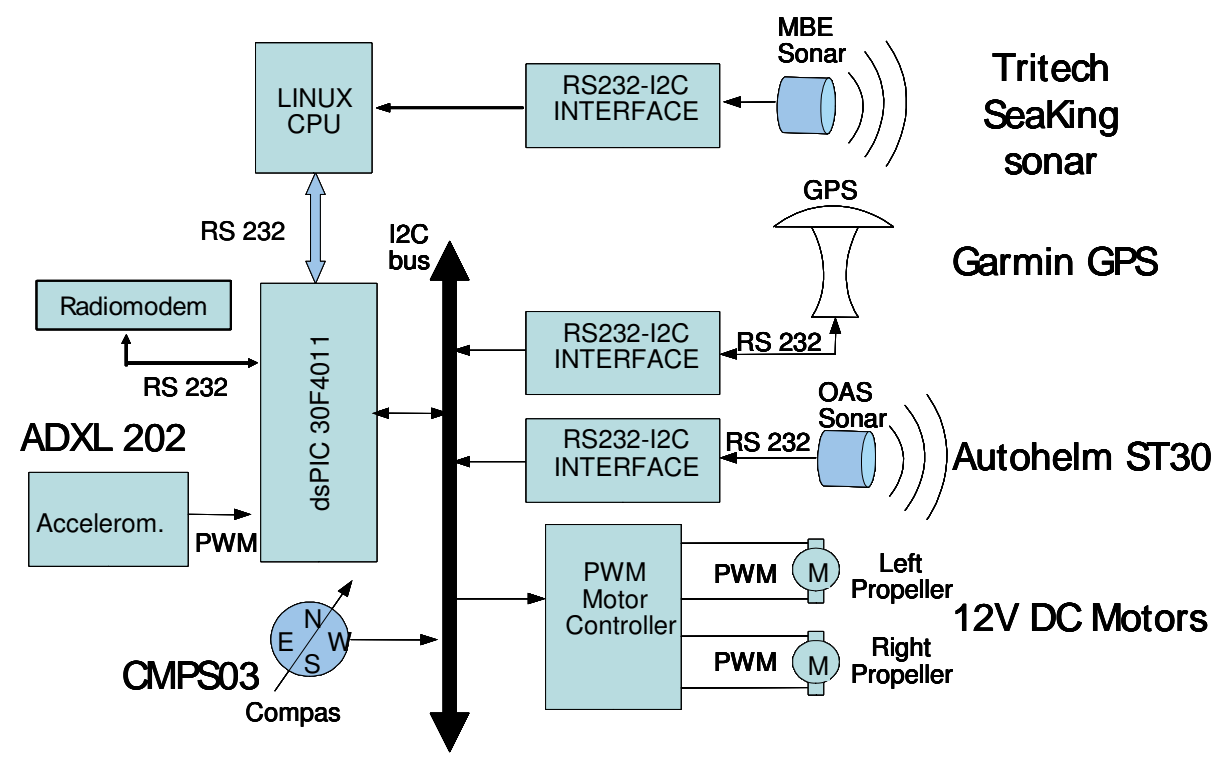

Fig. 3. The ICTIOBOT on-board electronics.

On the subject of the target tracking sensors, the MBE sonar is a Tritech Seaking and converts it readings into RS232 streams according to a proprietary data format. The obstacle avoidance sonar is an Autohelm ST30 depth finder and sends its echoes using Seatalk protocol (NMEA) by RS422 electrical signals that can be easily converted into RS232 signals 
Both the low level processor board and the high level board also communicate through RS232. Given the wide number of RS232 channels needed to interface all the modules and navigation sensors, it was decided to turn some of these signals into $\mathrm{I} 2 \mathrm{C}$ communication interface format. This popular and versatile bus used for communications among devices nodes inside equipments, can communicate with the slaves using different addresses and is easily configurable.

The digital compass is a CMPS03 robotics board based on the Philips KMZ51 magnetic field sensor, and is used to obtain the AUV orientation with respect to the earth magnetic field. This device, directly interfaced with the PC, provides a pulse width modulation (PWM) signal with the positive width of the pulse representing the angle apart of the north point. As regards the actuators, two propellers provide the horizontal movement with no need of rudder, and its DC motors are driven by a MD22 Dual H-Bridge device made by Robot Electronics capable to provide 50V-5A. The MD22 driver is based on a PIC microcontroller and accepts data communication over I2C bus. The vertical movement is provided by two propellants controlled by a MD03 50V - 5A driver from Devantech.

Finally, a link radio provides a safety mechanism to handle manually the AUV in the event of possible damages or flaws.

Any signal that comes from the transmitter at the frequency of $422 \mathrm{MHz}$ is considered as an interruption input to the microcontroller of the low level board, and consequently, stops attending the orders from the PC's communication serial port.

\subsection{Mechanical parts}

The low-cost ICTIOBOT prototype presented in figure 4 consists of two torpedoes assembled in an aluminium structure. These torpedoes are stimulated by two electrical propellants constructed by Motorguide and used by the divers as dragger vehicles. These are economic, support pressures of up to $6 \mathrm{~kg}$ and develop a speed of up to $2 \mathrm{mph}$ each one. They are done of glass fibber and take 4 batteries inside. The above mentioned batteries are of $12 \mathrm{~V} 33 \mathrm{AH}$ of absorbed electrolyte that give a good autonomy, considering that the tests of bigger duration and exigency reached 4 hours.

A high pressure canister is placed between both torpedoes and contains most of the mentioned electronics, except the compass and the communication systems, which are assembled apart in a watertight plastic box over the aluminium structure. The canister in turn is connected to other PVC box, everything using cable connectors that support pressure values associated with depths over $100 \mathrm{~m}$. The GPS, the high frequency modem and the WiFi adapter are protected by a water-repellent gel inside a few PVC's pipes.

The MBE is assembled in the front of the AUV structure and protected by a steel mask of a few aluminium bars that allow its vertical or horizontal assembly.

\subsection{Expert system based dynamic planner}

During an inspection, several unforeseen situations might appear like the detection by the FLS of a fishing net, or a complex pattern shape of more than one pipeline over the seabed recognized by the $\mathrm{MBE}$, or simply a detour due to obstacle detection. In these cases, it is hoped that the DMP module exhibits an "intelligent" behavior. To cope with these real situations in the marine world, it was resorted to the experience and skills of ROV operators. A little part of their knowledge was elicited and codified in the form of a real time expert 

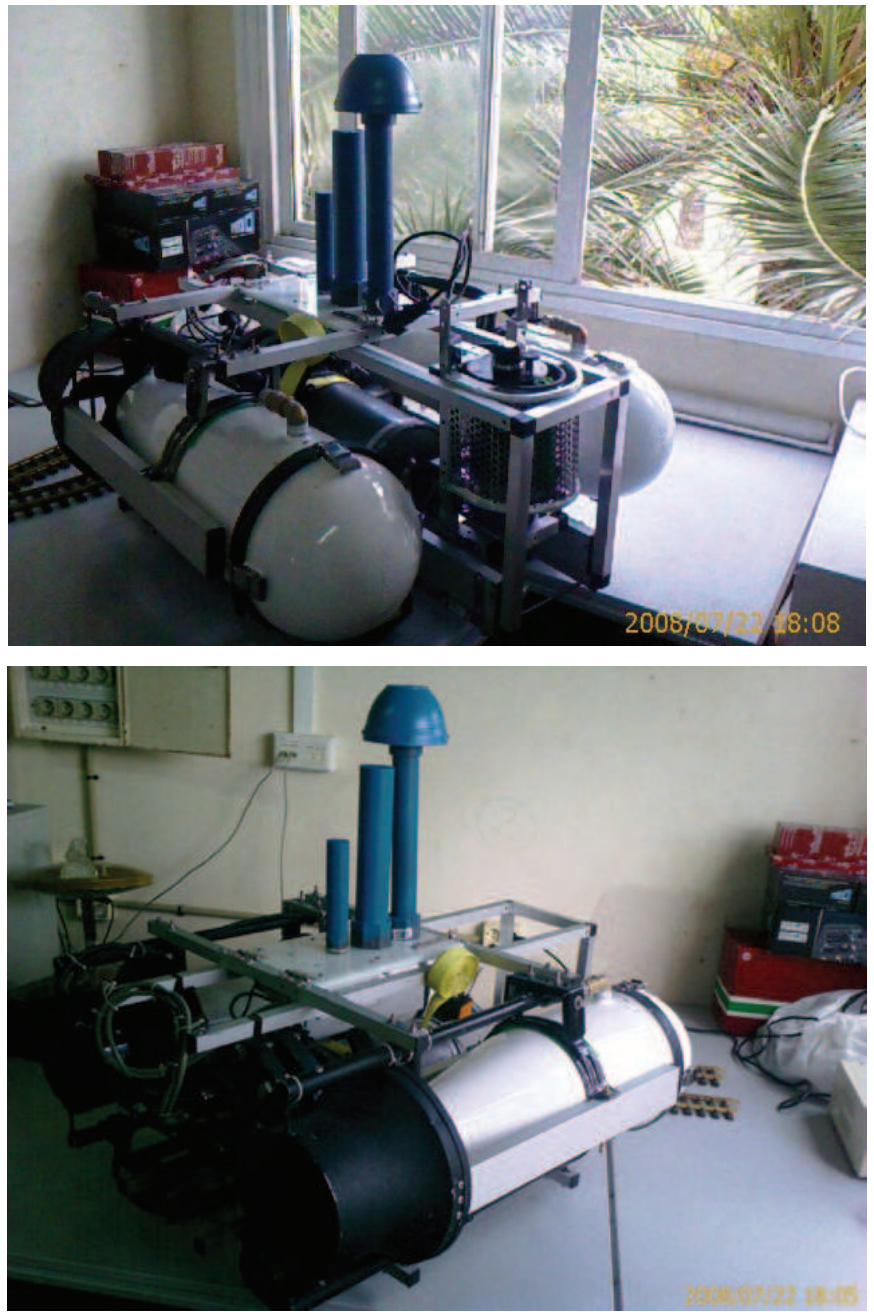

Fig. 4. Photographs of the low-cost ICTIOBOT prototype

system, the EN4AUV introduced earlier. It has been developed using CLIPS that constitutes a $\mathrm{C}$ language based shell, and allows the knowledge representation to be in the form of rules and frames (COOL or Clips Object Oriented Language). These formalisms are used in the knowledge base $(\mathrm{KB})$ to represent the involved knowledge. The main feature of the expert system is to assess a current situation in order to act accordingly, in a clearly data driven/reactive behavior. Thus, EN4AUV is a reactive expert system, taking the proper action for every different situation, and considering the pipeline/cable status, the type of survey, the different mission settings, and others.

These situations were coded as possible scenarios in about fifty rules, like the one presented in figure 5. As the knowledge about different situations increases, the knowledge base describing new scenarios can be completed an updated, yielding an incremental KB growth. 
Each scenario triggers different searching or tracking strategies, which are then subtasks with their own features. Scenarios are based mainly in two ideas: a) the survey type, and b) tracking states. The first one is defined a priori in the SMP module, to establish the number of pipeline/cables to be tracked, the navigation depth, and other mission features. The other basic component of the scenario determination is the tracking state that changes when the SFM module updates its sensors. From this information, the EN4AUV is able to decide the status of the pipeline/cable, that is, if buried, exposed, intermittent or free-span, and how is the AUV as regard as the pipeline/cable, that is, if avoiding an obstacle, with the object under study considered as found or lost, or returning to a previous known position.

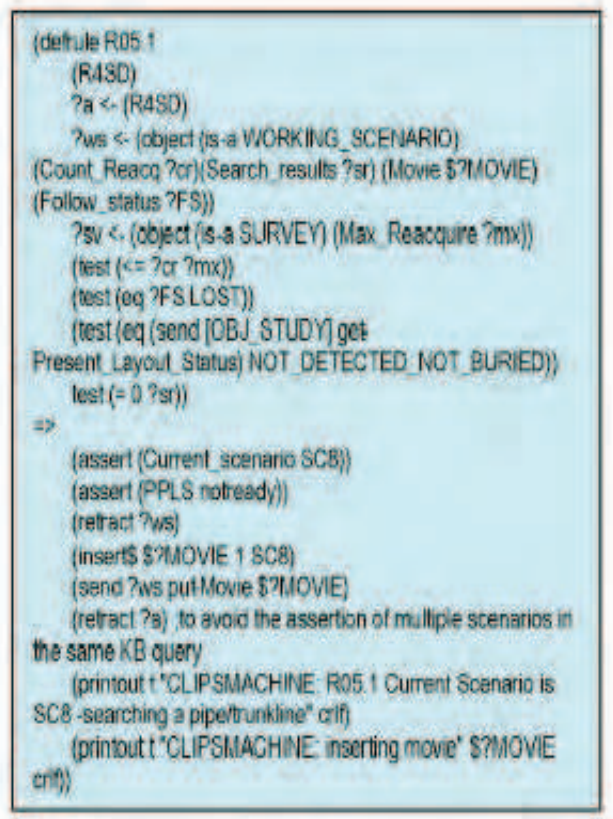

Fig. 5. A rule from the knowledge-base of the DMP for the $8^{\text {th }}$ scenario determination, in the typical CLIPS syntax

Once scenarios are established, a typical situation assessment task, EN4AUV must output a desired trajectory or must decide a pipeline/cable reacquisition.

To yield a desired trajectory, the actions are organized in a set o few simple subtasks: findstart, search, back to start, skip, and track. Then the final trajectory of the AUV is built by one of these subtasks, or by a concatenation of them. Examples of scenarios developed for the sea trials were the following ones:

- $\quad$ 1st Scenario: The AUV is tracking an exposed pipeline, navigating on top, at a fixed offset smaller or equal than 5 meters. Both the MBE and the MAG can detect it.

- 2nd Scenario: The AUV is tracking a buried pipeline on top, at a fixed offset, smaller or equal than 5 meters. The MBE may not be able to detect it, but the MAG can track it anyway.

- $\quad 3 r d$ Scenario: The AUV is tracking an intermittently exposed and buried pipeline at a fixed offset. This is a sequence of alternative appearance of scenarios number one and two. 
- 4th Scenario: The AUV is tracking a free-span pipeline at a fixed offset. The pipe is tracked mainly based on MBE readings, which may be detecting the pipe itself or the trench.

- 5th Scenario: The AUV is tracking a pipeline in the presence of one or more pipes (like infield pipelines) or other magnetic objects in the area. Measures from MBE as well as MAG are needed.

- $\quad$ 6th Scenario: The AUV is tracking a pipeline but avoiding an obstacle. In such scenario the certainty error may increase beyond its thresholds, but the EN4AUV knows where the pipe is and ignores the pipe_lost flag. The path planner module outputs a flag indicating this condition and the EN4AUV may query the legacy data to confirm the existence of an exclusion zone. Although sensor readings are not reliable, they are not turned off to be ready when the AUV is again over the pipeline.

- $\quad$ 7th Scenario: The AUV is searching a buried pipeline. No readings from MBE, just MAG will yield detection when the AUV is right over the pipe. With two detection (crossing) points the pipeline direction vector is computed and the AUV starts tracking from the last known point with this direction.

- $\quad$ 8th Scenario: The AUV is searching the pipeline, which is considered as lost. EN4AUV shall have an estimate of the trajectory from SFM considering the whole inputs: MBE, MAG, and LD. If not, it will estimate the initial parameters from mission settings and LD.

- 9th Scenario: The AUV is searching a pipeline in the presence of one or more pipes (like infield pipelines) or other magnetic objects in the area. Every information source is operative to discriminate the target under study (MBE, MAG, and LD).

- 10th Scenario: The AUV is skipping from one point to another. MBE, and MAG are off to save energy. This special situation appears when changing from one pipe to another to track, or from one zone of interest to another over the same pipeline.

- 11th Scenario: The AUV is going back to the last known position to start tracking, after founding the pipeline as a consequence of a successful search. MBE, and MAG are off.

- $\quad$ 12th Scenario: The maximum number of reacquisition after unsuccessful searches was reached. The mission is ended with a failure message.

- 13th Scenario: The AUV is tracking an exposed pipeline, navigating on top, at a fixed z_offset greater than 5 meters. The detection is done mainly with the MBE.

- $\quad$ 14th Scenario: The AUV is tracking a buried pipeline on top, at a fixed z_offset greater than 5 meters. The blind tracking is done mainly based on legacy data, and cannot last more than half a minute. After this, if there are no more sensor readings, a new search must be started.

\subsection{Development and runtime environments}

A leading idea was to employ the same environment for the software development than for the runtime, with a minimum adaptation time for porting it from one to the other. The subsystems were first tested in simulation, within a development environment named ATMEnvs (Curti et al., 2005) such that the software could be directly ported to the vehicle when these simulation tests were successful. Thus, the simulation environment was communicated with the vehicle software through the serial port, using an adaptor between the NMEA protocol and the messages within a proprietary communication protocol among modules. In this way, once the software is operative, the simulator is easily and 
straightforward replaced by the real hardware prototype. The simulation environment included cinematic and dynamic linear models of the vehicle. The static information among the different modules was shared through bottom specification files ( ${ }^{*}$.bsf) and objective under inspection specification files (*.psf). The information associated with the dynamic variables among the modules travel in the messages mentioned before, the same that is employed when the robot is operating in its real environment.

Referring to figure 2 in Section 3.2, the DMP and OAS constitute a system in cascade connection; consequently, if the OAS did not detect any object through the FLS, its output will be simply the desired trajectory from the DMP. On the contrary, if an obstacle is detected, the OAS changes the necessary waypoints in the trajectory provided by the DMP.

\section{Experimental results}

Many approaches were implemented and tested for the control system. For instance a linear PI-MIMO (Proportional Integrative - Multiple Input Multiple Output) plus feed-forward structure (Valenciaga et al., 2007) and also a nonlinear fuzzy logic based control were verified in computer simulation. They were applied in a low-cost ocean observing platform, based on a hybrid vehicle between an AUV and autonomous surface vehicle (ASV), called CORMORAN. This robot moves slightly underneath the sea surface following a previously planned route and regularly dives to make vertical profiles of the water column. The PIMIMO control strategy is used to commands the rudder and the propeller.

However, the control system currently mounted in ICTIOBOT is a simple PI-MIMO control in the horizontal plane (2D) minimizing the angle $\beta$, that is given by Equation 1 .

$$
\beta=\lambda-\Psi
$$

where $\Psi$ is the vehicle's yaw and $\lambda$ the angle given by Equation 2, where $\left(x_{1}, y_{1}\right)$ and $\left(x_{2}, y_{2}\right)$ the coordinates of the robot and the target waypoint, respectively (figure 6). This is known as the line-of-sight (LOS) guidance law (Naeem et al., 2003). The speed envelope using an ideal particle approach, recently proposed (Breivik and Fossen, 2005) was also tested in computer simulation (Valenciaga et al., 2007), but not yet ported to the prototype.

$$
\lambda=\tan ^{-1}\left(\frac{y_{2}-y_{1}}{x_{2}-x_{1}}\right)
$$

The navigation system for the preliminary trials showed in this chapter, consisted on a sensor fusion of measures from a compass, an inertial navigation system, a depth sensor and a GPS (when navigating in surface), for self position perception. For pipeline tracking, the prototype used an $\mathrm{MBE}$, to avoid the need of light for image processing near the dark seabed when water is not clear or the applications shift to deeper waters.

The knowledge-based DMP was also integrated to the ICTIOBOT prototype. The evolutionary robotic OAS was not ready to be tested in these trials. Several tests were carried out in the sea surface and slightly underneath the surface, to have available the signal from GPS in all the cases.

These modules were tested in the trials done in Cala Estancia that is bay with scanty meters of depth, near to Palma de Mallorca, Spain, in April 2008. A curved trajectory was the reference of the step points, represented by solid red line in figure 7 , whereas the punctuated line in the same figure represents the real position described by the AUV. 


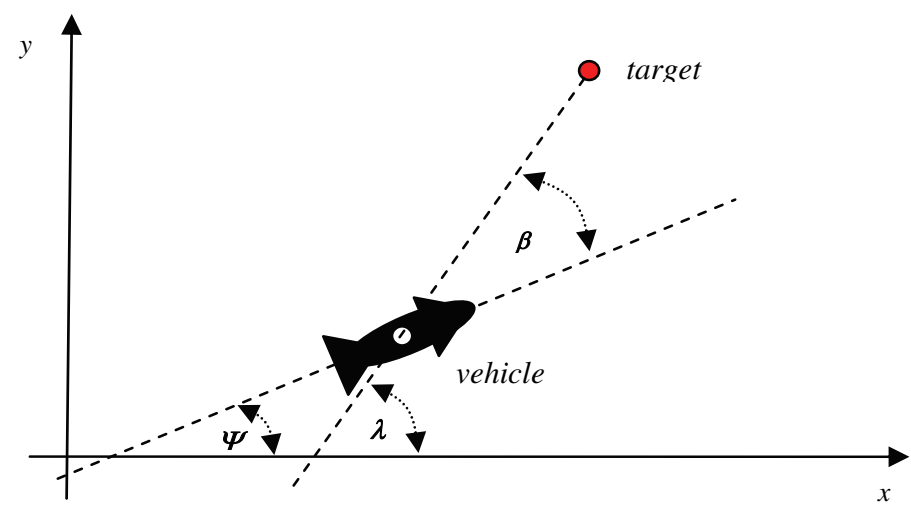

Fig. 6. Line of sight (LOS) guidance approach.

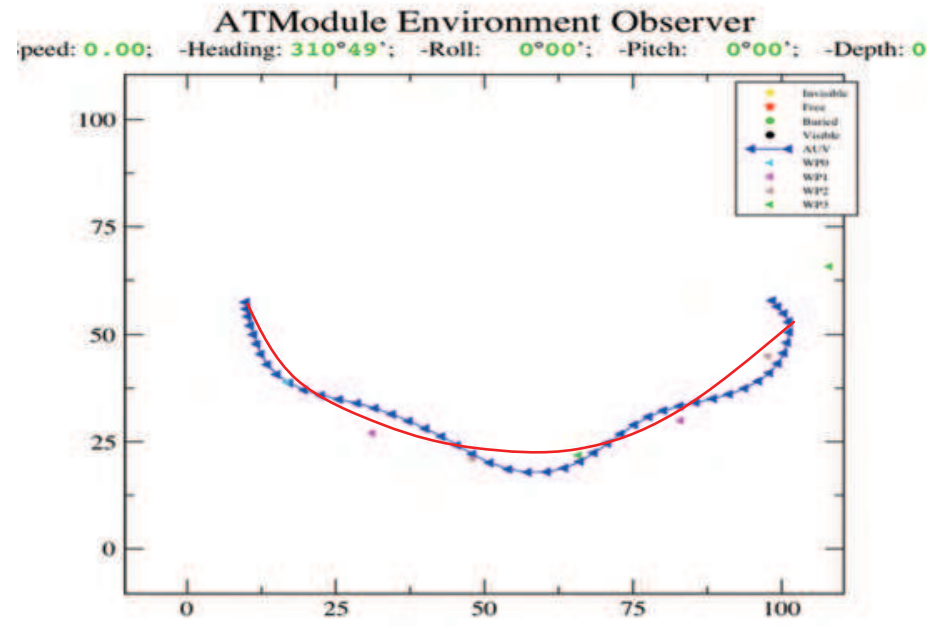

Fig. 7. Test of the ICTIOBOT prototype, in slightly deep waters of the coast of Mallorca, Spain, tracking a curved trajectory

As it can be seen, navigation, guidance and control systems are very elementary and even in these calm waters the trajectory presents severe deviations from the reference.

A second set of trials was carried out with a pipe in the seabed, painted in yellow in figure 8 . As it may be seen, the prototype begins in the position (58.0), relative to the trial location, and moves to the position (65.25) to initiate the search. When this point is found, after doing a trajectory in shape of eight, the sonar is turned off in order to simulate a search strategy proposed by the DMP.

Thus the reference appears in zigzag for the wished path. This trajectory is depicted by step points which were united by a solid red line for better clarity. Again, the necessary improvement is demonstrated in the guidance and control systems, since with the bigger inertia on account of the weight of the sonar and the associate electronics, the AUV moves away from the reference imposed by the DMP. 


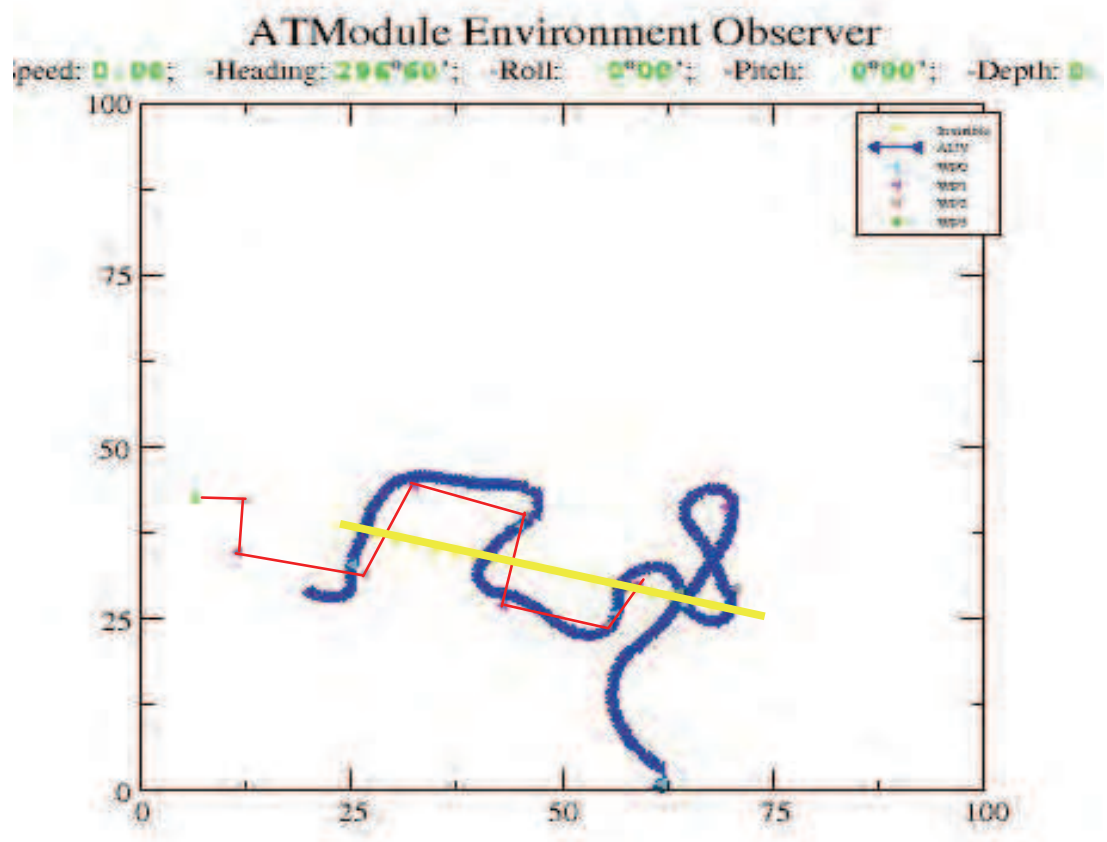

Fig. 8. Test in Mallorca, Spain; looking for a pipeline

\section{Conclusion}

The key aspects of the software and hardware elements design for an AUV prototype have been presented, as a low purchasing and operation cost adaptation of other vehicles over which authors have been working in other projects. In particular, the dynamic mission planner based on an artificial intelligence paradigm showed to be very suitable to be reused in different robots with different inspection missions. This mission planner showed that this approach is able to re-plan the vehicle trajectory while in the mission, taking into account the original static mission settings, the changing underwater environment and the situation of the target under inspection. The new experimental prototype ICTIOBOT presented here will surely be an adequate test-bed for the new task and path planning algorithms. In particular we will focus in a near future in the enlargement of the knowledge-base within the DMP for untested use-cases.

The work also shows that it is possible with current technology to construct a low-cost AUV for pipeline and cable tracking that can be used for preventive maintenance purposes of submarine infrastructure. In addition, the prototype is useful for test and improvement of each module separately.

From the obtained results is possible to observe that there is still a need to improve several aspects in the guidance, control and navigation systems, since the AUV's real path moves away considerably from the desirable trajectory proposed by the DMP module. 


\section{Future work}

It will be proposed different approaches for each of the described subsystems: the Dynamic Mission Planner, the Guidance System, the Control System and the Navigation System. They will be evaluated and verified on the experimental prototype.

For the DMP, it is expected to add new rules for describing more complex scenarios and cases required by the users as, for instance, multiple pipelines tracking, recognition of different pattern shapes like fishing nets, mines, anchors, and wastes nearby to the pipeline. To achieve this it is proposed a devoted sensor that uses the sonar information for filtering it by means of an artificial neural network and operates as a special input to the knowledge base. For the Guidance and Control Systems, it is expected to continue searching which is the best approach for the problem to be resolved. Specially, is desirable that the system that finally controls the AUV is of the adaptive type in order to minimize possible errors that can appear due to on-board variable payload. For the Navigation System, after studies and experimental tests it will be selected a method to implement a sensor fusion system to determine the proper AUV position and attitude, with a bounded accuracy. Based on this information it will be possible to obtain the target positions and recognized patterns in the seabed. Among the possible approaches to be analyzed, the most promising seems to be Kalman's filters and those who combine sensor inputs using fuzzy logic techniques. It is also expected that a side-scan sonar and a magnetic sensor for target tracking will be added in future stages of the project to enhance the possibilities of target and obstacle perception of the AUV.

\section{Acknowledgments}

The research and development work described in this chapter was possible due to the financial support of the following projects: TRA2006-13318 MEC (Spain), PCI2005-A7-0356 FEDER (EU), AUVI-MIF2-CT-2004-003027, FP6 (EU), and PAE 22696 National Agency ANPCyT (Argentina).

\section{References}

Acosta, G. G. ; Calvo Ibáñez, O. A. ; Curti, H. J. \& Rozenfeld A. F. (2007). Low cost autonomous underwater vehicle for pipeline and cable inspection, Proceedings of IEEE UT07/SSC07 - Underwater Technology 2007 and Workshop on Scientific Use of Submarine Cables \& Related Technologies 2007, Vol. 1, pp. 331-336, ISBN: 1-4244-12080/07, Tokyo, Japan, April 17-20 2007.

Acosta, G. G. ; Curti, H. J. ; Calvo, O. A. \& Rossi, S. R. (2006). A knowledge-based approach for na AUV path planner development. WSEAS Transactions on Systems, Vol. 5, No. 6, June 2006, pp. 1417-1424, ISSN: 1109-2777.

Acosta, G. G. ; Curti, H. \& Calvo, O. (2005). Autonomous underwater pipeline inspection in AUTOTRACKER PROJECT : the navigation module, Proceedings of IEEE-OCEANS'05 Europe Conference, Vol. 1, pp. 389-394, Brest, France, June 21232005. 
Acosta, G. G. ; Curti, H. ; Calvo, O. \& Mochnacs, J. (2003). An Expert navigator for an autonomous underwater vehicle, Proceedings of SADIO/Argentine Symposium on Artificial Intelligence ASAI'03, in CD, Buenos Aires, Argentine, September 2003.

Antonelli, G.; Chiaverini, S.; Finotello, R. \& Schiavon, R. (2001). Real-time path planning and obstacle avoidance for RAIS: an autonomous underwater vehicle. IEEE Journal of Oceanic Engineering, Vol. 26, No. 2, April 2001, pp. 216-227, ISSN: 0364-9059.

Balasuriya, A. \& Ura, T. (1998). Autonomous target tracking by underwater robots based on vision, Proceedings of IEEE International Symposium on Underwater Technology, pp. 191-197, Tokyo-Japan.

Barron, J. L.; Fleet, D. \& Beauchemin S. (1994). Performance of optical flow techniques. Int. Journal of Computer Vision, Vol. 12, No. 1, pp. 43-77, ISSN: 0920-5691.

Bartumeus, F.; Catalan, J.; Fulco, U. L.; Lyra, M. L. \& Viswanathan G. M.. (2002). Optimizing the encounter rate in biological interactions: Lévy versus Brownian strategies. Physical Review Letters, Vol. 88, No. 9, March 2002, pp. 1-4, ISSN: 0031-9007.

Breivik, M. \& Fossen, T. (2005). A unified concept for controlling a marine surface vessel through the entire speed envelope, Proceedings of IEEE Mediterranean Conference on Control and Automation, pp. 1518-1523, Limassol, Cyprus, June 27-29 2005.

Curti, H. ; Acosta, G. G. \& Calvo, O. (2005). Autonomous underwater pipeline inspection in AUTOTRACKER PROJECT : the simulation module, Proceedings of IEEE-OCEANS'05 Europe Conference, Vol. 1, pp. 384-388, Brest, France, June 21232005.

Evans, J. ; Petillot, Y. ; Redmond, P. ; Wilson, M. \& Lane, D. (2003). AUTOTRACKER : AUV embedded control architecture for autonomous pipeline and cable tracking, Proceedings of IEEE-OCEANS 2003, Vol.5, pp. 2651-2658.

Fernández León, J. A.; Acosta, G. G. ; Mayosky, M. A. \& Calvo Ibáñez, O. (2008). A biologically inspired control based on behavioural coordination in evolutionary robotics, Chapter in: Advancing Intelligence through Biological Process Applications, Idea Group Inc., pp. 107- 129, ISBN: 978-1-59904-996-0.

Fernández León, J.; Tosini, M \& Acosta, G. G. (2004). Evolutionary reactive behavior for mobile robots navigation, Proceedings of 2004 IEEE Conference on Cybernetics and Intelligent Systems (CIS04), pp. 532-537, Singapore, December 1-3, 2004.

Looney, C. (1997). Pattern Recognition Using Neural Networks, Oxford University Press, ISBN: 0-19-507920-5, Oxford/NY.

Naeem, W.; Suttom, R. ; Ahmad, S.\& Burns, R. (2003). A review of guidance laws applicable to unmanned underwater vhicles. The Journal of Navigation, Vol. 56, pp. 15-29

Rives, P. ; Breuil, E. \& Espiau, B. (1986). Recursive estimation of 3D features using optical flow and camera motion, LAS, pp. 522-532.

Shi, J. \& Tomasi, C. (1994). Good features to track, Proceedings of IEEE Computer Soc. Conference on Computer Vision and Pattern recognition (CVPR'94), pp. 593-600, Seattle, USA, June 1994. 
Valenciaga, F. ; Puleston, P. F. ; Acosta, G. G. \& Calvo, O. (2007). Trajectory tracking of the Cormoran AUV based on a PI-MIMO approach, Proceedings of IEEE-OCEANS'07 Europe Conference, pp. 1-6, ISBN: 978-1-4244-0635-7, Aberdeen, Scotland, June 18-21 2007. 


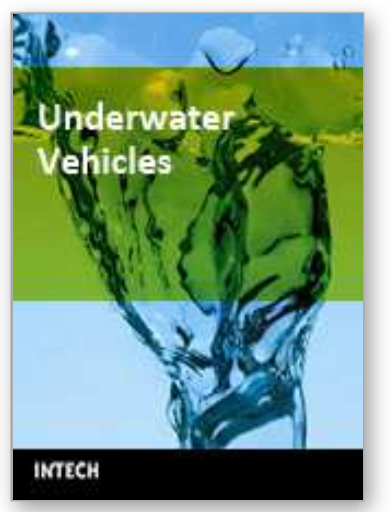

\author{
Underwater Vehicles \\ Edited by Alexander V. Inzartsev
}

ISBN 978-953-7619-49-7

Hard cover, 582 pages

Publisher InTech

Published online 01, January, 2009

Published in print edition January, 2009

For the latest twenty to thirty years, a significant number of AUVs has been created for the solving of wide spectrum of scientific and applied tasks of ocean development and research. For the short time period the AUVs have shown the efficiency at performance of complex search and inspection works and opened a number of new important applications. Initially the information about AUVs had mainly review-advertising character but now more attention is paid to practical achievements, problems and systems technologies. AUVs are losing their prototype status and have become a fully operational, reliable and effective tool and modern multi-purpose AUVs represent the new class of underwater robotic objects with inherent tasks and practical applications, particular features of technology, systems structure and functional properties.

\title{
How to reference
}

In order to correctly reference this scholarly work, feel free to copy and paste the following:

Gerardo Gabriel Acosta, Hugo Curti, Oscar Calvo Ibáñez and Silvano Rossi (2009). Some Issues on the Design of a Low-Cost Autonomous Underwater Vehicle with an Intelligent Dynamic Mission Planner for Pipeline and Cable Tracking, Underwater Vehicles, Alexander V. Inzartsev (Ed.), ISBN: 978-953-7619-49-7, InTech, Available from:

http://www.intechopen.com/books/underwater_vehicles/some_issues_on_the_design_of_a_lowcost_autonomous_underwater_vehicle_with_an_intelligent_dynamic_mi

\section{INTECH}

open science | open minds

\author{
InTech Europe \\ University Campus STeP Ri \\ Slavka Krautzeka 83/A \\ 51000 Rijeka, Croatia \\ Phone: +385 (51) 770447 \\ Fax: +385 (51) 686166 \\ www.intechopen.com
}

\author{
InTech China \\ Unit 405, Office Block, Hotel Equatorial Shanghai \\ No.65, Yan An Road (West), Shanghai, 200040, China \\ 中国上海市延安西路65号上海国际贵都大饭店办公楼405单元 \\ Phone: +86-21-62489820 \\ Fax: +86-21-62489821
}


(C) 2009 The Author(s). Licensee IntechOpen. This chapter is distributed under the terms of the Creative Commons Attribution-NonCommercialShareAlike-3.0 License, which permits use, distribution and reproduction for non-commercial purposes, provided the original is properly cited and derivative works building on this content are distributed under the same license. 